\title{
Methods in Cognitive Neuroscience: A primer for Forensic Psychologists
}

\section{Hedwig Eisenbarth}

To cite this article: Hedwig Eisenbarth (2018): Methods in Cognitive Neuroscience: A primer for Forensic Psychologists, Psychology, Crime \& Law, DOI: 10.1080/1068316X.2018.1425409

To link to this article: https://doi.org/10.1080/1068316X.2018.1425409

Accepted author version posted online: 08 Jan 2018.

Submit your article to this journal $₫$

Q View related articles $₫$

View Crossmark data 
Publisher: Taylor \& Francis \& Informa UK Limited, trading as Taylor \& Francis Group Journal: Psychology, Crime \& Law

DOI: 10.1080/1068316X.2018.1425409

Methods in Cognitive Neuroscience: A primer for Forensic Psychologists

Hedwig Eisenbarth

University of Southampton

Running head: COGNITIVE NEUROSCIENCE METHODS

Corresponding author:

Hedwig Eisenbarth

Department of Psychology

University of Southampton

University Road

SO17 1BJ

Southampton, UK

h.eisenbarth@soton.ac.uk 


\title{
Methods in Cognitive Neuroscience: A primer for Forensic Psychologists
}

\begin{abstract}
Cognitive Neuroscience provides a rich variety of methods for the investigation of the neural underpinnings of human behaviour. This paper provides an overview of the most popular measurement methods used in neuroscience such as EEG and MRI, that have relevance for forensic psychology. After outlining these methods, I provide some examples from forensic research. The paper closes with a brief discussion of the challenges confronting forensic researchers and clinicians using neuroimaging in research as well as in the court room.
\end{abstract}

Keywords: Imaging, fMRI, EEG, ERP, Cognitive, Forensic 


\section{Methods in Cognitive Neuroscience: A primer for Forensic}

\section{Psychologists}

The breadth of neuroscientific assessment and analysis methods for cognitive processes has been growing exponentially through the last decades and a delayed but similar growth of research on forensic questions can be observed. A Web of Science (https:// webofknowledge.com/ ) search using the search terms fMRI/ EEG and criminal/ antisocial shows the steady increase in publications since $2000(1,305$ publications) and a plateau reached since 2014 (6,173 publications). At the same time as the use of neuroscience methods in research in forensic psychology rose, the question about the use of neuroscience methods in the courtroom adds to the relevance of understanding the methods and related reported findings, linked to terms like Neurolaw and Neurocriminology (see e.g. Choi, 2017; Glenn \& Raine, 2014). Thus, this paper discusses the methods of neuroscience that are relevant for forensic psychology for both perspectives: understanding their use in the context of research on pathways to criminal and antisocial behaviour, and in the context of forensic assessments. It reviews first structural measures, then functional measures of brain imaging, with a specific emphasis on the most common measures such as EEG and fMRI, including example studies for each method. This is followed by a brief overview of potential challenges for the use of neuroscientific methods in research and in the court room.

\section{Imaging the Brain}

Brain imaging techniques have become important tools in the investigation of the neurophysiological correlates of behaviour. These imaging techniques can be divided into those that provide structural information and those that supply functional information. The former group includes Magnetic Resonance Imaging (MRI) as well as Computed Tomography (CT). The latter can be differentiated by 
what neurophysiological processes they rely on as well as by time versus spatial resolution (see Table 1). Electrophysiological processes can be measured using e.g. Magnetoencephalography (MEG) or Electroencephalography (EEG), which differ in terms of their time and spatial resolution. While metabolic processes can be measured with functional Magnetic Resonance Imaging (fMRI), Diffusion Tensor Imaging techniques (DTI) or Positron Emission Tomography (PET). For an overview of functional methods, see Table 1.

insert table 1 about here

\subsection{Structural measures}

Magnetic Resonance Imaging (MRI) uses a strong magnetic field to create images based on changes of the alignment of hydrogen molecules, with different types of tissues (e.g. varying signals based on white or grey matter of the brain). These images can be used for structural analyses (e.g. Voxel Based Morphometry - VBM), tractography (e.g. Diffusion Tensor Imaging - DTI), and functional analyses (i.e. functional MRI - fMRI).

Voxel Based Morphometry (VBM) allows a quantification of the volume of grey yersus white matter and cerebrospinal fluid in an automated way (for an overview see: Bigler, 2015). VBM maps images taken by MRI to a template, smoothens images so that each voxel represents the average of the voxel and surrounding voxels and then compares the image volume across the brain for every voxel. As the resulting volumes can vary between different at different time points within the same person, it has to be taken into account, what the participant is doing during the rest period in which images are taken. However, it is the most common 
approach to the investigation of structural brain differences and has been widely applied in forensic research.

Potential application: For example, a recent systematic review on structural brain abnormalities in interpersonal violence reports across 35 studies a significant reduction of grey matter in the parietal lobe in perpetrators, however the related voxel based meta-analysis across 6 studies did not find significant differences (Lamsma, Mackay, \&Fazel, 2017). On the other hand, a study that was using VBM derived data, could accurately distinguish adolescent offenders for high versus low scores of psychopathic traits (Steele, Rao, Calhoun, \& Kiehl, 2017). The brain structures that were implicated in this differentiation were paralimbic structures such as the Right Parahippocampus, Posterior Cingulate Cortex, as well as Left and Right Temporal Pole, which are regions that have been linked to executive control (Calhoun, Adali, Pearlson, \&Pekar, 2001). Thus, in this example, brain structural information seems to be different enough to be able to differentiate between juvenile offenders with high and low psychopathic traits. The potential for diagnostic value is going to be discussed at the end of this review.

Diffusion Tensor Imaging (DTI) is a structural or functional measure, as it is based on structural data, but allows for interpretation of functional aspects. It is based on measuring the rate of tissue water diffusion and therefore can be interpreted to describe white matter tracts in the brain (Alexander, Lee, Lazar, \& Field, 2007).

Potential applications: A recent review on the relationship between diffusivity of white-matter tracts and antisocial behaviour across development covers 22 studies, which show a positive correlation between antisocial behaviour and diffusivity for several tracts: the uncinate fasciculus, inferior fronto-occipital 
fasciculus, cingulum, corticospinal tract, thalamic radiations, and corpus callosum (Waller, Dotterer, Murray, Maxwell, \& Hyde, 2017).

Another analysis method based on MRI derived data is the Query, Design, Estimate, Contrast (QDEC) analysis, which is used to investigate cortical thickness. Understanding the altered development of the "antisocial brain" can not only be investigated by volumes of certain brain areas or white matter tracts, but also by adding the variable cortical thickness.

Potential applications: For example, a study by Oostermeijer and colleagues (2016) in a community based youth sample found that those adolescents with desisting conduct problems exhibited less reduction of cortical thickness in the dorsolateral prefrontal cortex and the anterior cingulate cortex as well as the hippocampus, and an increase of thickness in the hippocampus, compared to a group with low conduct problems.

\subsection{Functional measures}

Functional brain imaging measures use metabolic processes in order to gain insight into activity of brain areas, using the assumption that a higher metabolic rate in a specific area, the higher the activity of that area. The most commonly used functional imaging measures with relevance for forensic psychology are, as mentioned above, EEG, fMRI and PET, which will now be explained.

\subsubsection{Electroencephalography (EEG)}

Measurements of electrocortical signals via EEG are still the method of choice for investigations of fast cortical processes and for research questions that need a detailed time resolution. EEG is a non-invasive technique measuring the voltage fluctuations from ionic current in the neurons via sets of electrodes placed on the scalp (see Figure 1). EEG allows to detect changes in electrical activity almost in real time, tracking the time course of neural events within 20-100ms. Obtaining EEG 
data requires the appropriate equipment (a standard EEG cap uses 64-120 channels), training in its use, and ideally an assessment environment that is electrically noise-free or reduced (Keil et al., 2013). Recently, the development of less expensive and wireless versions, which usually come with less channels have been tested and found to provide data of similar reliability compared to the full channel wired version, at least for one specific attentional task (Barham et al., 2017).

The EEG signal can be obtained during rest or in the context of different types of stimulation, e.g. presented tasks or stimuli. Resting EEG signals are usually assessed for the cumulative presence of certain frequencies, while task-based EEG signals can be analysed not only by frequency domain (1), but also by time domain (2) or by the combination of time and frequency (3).

\subsubsection{Frequency domain based EEG}

Frequency domain analyses allow researchers to understand proportions of frequencies present in given time periods in the investigation of psychological processes such as information processing (e.g., Zhong et al., 2017) or different states of consciousness (REF). Frequencies are usually described by ranges: beta ( $>13 \mathrm{~Hz}$ ), alpha (8-13 Hz), theta (4-8 Hz) and delta $(0.5-4 \mathrm{~Hz})$, with the alpha frequency the most known frequency range, related to relaxed states (see Figure 1).

insert figure 1 about here

Apart from the proportional presence of different frequencies at a given time, other effects like local distribution of those frequencies or asymmetries between cortical hemispheres can be investigated. These data usually are interpreted by comparing frequency presence across different groups of individuals. 
Potential applications: An example for the use and interpretation of frequency distributions is the study by Reyes and Amador (2009), which compared EEG derived frequencies of offenders with Antisocial Personality Disorders (ASPD) with those offenders without ASPD during rest. They found a reduced alpha rhythm as well as an increased amount of theta and delta frequencies in frontal areas in the ASPD group. The authors interpret this as evidence for altered motivational and inhibitory states in criminal offenders, which is more pronounced in those with ASPD. As alpha rhythm has been linked to inhibitory processes and theta have been linked to inhibitory control these results point to a dysbalance in these frequencies being linked to behaviour control such as aggressive behaviour control.

A by now rather robust finding is the correlation of frontal asymmetry of the alpha band during rest with trait measures of inhibition, with reduced right frontal activity correlates with higher impulsivity (for a review see: Harmon-J ones, Gable, \& Peterson, 2010; e.g., Neal \& Gable, 2017). The correlation between self-reported impulsive aggression and frontal activity independent of frequencies has been not only been found in individuals with a criminal history, but also in undergraduate samples: students who self-reported impulsive aggressive behaviour showed a reduced right frontal alpha activity and relatedly an increased frontal asymmetry (Lake, Stanford, \&Patton, 2014). This replication of the reduced alpha activity related to impulsive aggression in conjunction with an increased asymmetry, points to a physiological correlate of aggressive behaviour. This not only links aggressive behaviour to frequencies that have been consistently found to be relevant for behaviour control, but furthermore provides implications for potential treatment interventions. Although at this stage electrophysiology based treatments such as neurofeedback have only limited evidence (Fisher, 2007; Konicar et al., 2015), they should be investigated in future work. In addition, frontal asymmetry has been 
linked to sexual motivation (Prause, Staley, \&Roberts, 2014), however, this has not yet been investigated in criminal samples. Thus, not only prevalence of certain frequencies but also balance of frequencies across different brain areas seem to match existing basic research on behaviour control more importantly being linked to impulsive aggressive behaviour on a day-to-day basis, confirming the relevance of training of behaviour control as an important target for impulsive aggressive individuals.

\subsubsection{Time domain based EEG}

Another approach, which allows more specific analyses, e.g. in conjunction with stimulus reactivity is based on time domain. Time domain based analyses are mainly referring to event-related potentials (ERP), which depict the average activity at certain electrode sites or groups of electrodes after some sort of stimulation (fugre 2 for an overview and figure 3 for an example). Activity related to an event, e.g. the onset of a facial expression (starting at oms in the plot presented in Figure 2) shown to the participant on the screen can be compared to activity in control conditions (e.g. facial expression of a different emotional valence) or to the activity another group of individuals presents (e.g. comparing individuals with different levels of psychopathic traits).

insert figure 2 and 3 about here

The characteristics of the average activity are actually providing the labels for these ERPs, with changes in positive direction from baseline indicated with "P" and negative changes indicated by “ $N$ ”; both can be followed with numbers which refer to the time after stimulus onset, e.g. an N1 or N100 reflects a negative component 100ms after stimulus onset (see figure 2) (for a detailed overview see: Picton et al., 
2000; Woodman, 2010). Further, ERPs have been established by paradigms or specific cues. For example, errors, such as the Error Related Negativity (ERN), a negative component that can be found about 50ms after committing an error (Olvet \&Hajcak, 2009) or the Early Posterior Negativity (EPN) as a negative time domain component that can be found about round 150-300ms after stimulus onset and seems to be characteristic for early attentional processing of emotional content and reflect the degree of arousal (Schupp, Flaisch, Stockburger, \&J unghoefer, 2006). Table 2 provides a rough overview of the most common ERPs and some of their suggested related processes and figure 2 shows the timing of the related components.

\section{insert table 2 about here}

Potential applications: As this method allows to investigate neural processing of events such as perception of emotional cues or committing an error, numerous studies have investigated this type of cue processing in the context of antisocial behaviour or sexual interest. Common cognitive paradigms applied for this type of investigation range from picture viewing to reaction time based tasks like Go/ Nogo-tasks or Oddball tasks. One very common ERP is the P3 within behaviour inhibition tasks. Gao and Raine (2009) provided a meta-analysis on the P3 alteration specifically in ASPD and psychopathy across 38 studies, finding reduced P3 amplitude and latency in different cognitive tasks (e.g. Oddball tasks, Go/Nogo tasks, Selective attention tasks and for both visual and auditory stimuli) correlated with antisocial behaviour, independent of control group type. However, based on the type of studies included in that meta-analysis no effect of psychopathy was found. The conclusion the authors draw is that antisocial behaviour might be reflected in an altered connectivity of frontal and temporal regions of the brain, which are related in 
updating and memory access, which are subsequently relevant for decision making. A recent publication investigated the modulation of the P3 (see Figure 4) by callousunemotional traits (Gao et al., 2018) and found a reduced P3 to be linked with the combination of interpersonal and behavioural aspects of callous-unemotional traits in youth. This not only re-iterates the relevance of a reduced P3 for the behavioural and interpersonal aspects of ASPD, but also shows that this physiological correlate of reduced reaction to novel stimuli might explain behavioural problems in these individuals by a suggested reduced interaction between frontal and temporal areas of the brain. Understanding these processes better could in the future contribute to early detection of problematic stimulus processing as well as providing basis for the development of potential interventions targeting these processes.

Another example for the use of ERPs in forensic research are correlates of sexual interest. Although it has not yet been used in criminal populations, findings from studies in the general population point to interesting applications in sexual offenders: for example, the Late Positive Potential (LPP) has been linked to the number of intercourse partners (Prause, Steele, Staley, \& Sabatinelli, 2015) as well as the P3 to sexual arousal in men and the N1 in women (Oliver, Meana, \& Snyder, 2016).

Important to note at this point, that the examples provided so far are all based on correlational approaches. Thus, they contribute to the understanding of potentially altered processing. However, in conjunction with findings from research using other brain imaging methods, e.g. MRI and fMRI, their interpretation can be broader and provide basis for the development of evidence based models explaining antisocial behaviour (see Ward, Wilshire \&J ackson, this issue).

\subsubsection{Functional MRI}


The most commonly used approach to the understanding of brain functions is functional MRI (fMRI), which uses MRI derived Blood Oxygen Level Dependent (BOLD) signal data. It capitalizes on the idea that areas that are more active also show more blood flow and therefore it contrasts in-homogeneities in the magnetic field due to changes in the level of oxygen in the blood. These images (depending on the specifics of the instrument and the scanning parameters 1-6 per 3s) can then be compared across different study conditions. For example, neutral versus emotional pictures presented or analysed during a rest period (resting state). Common approaches are contrasts (comparing different task conditions or groups) for regions of interest (ROI) or whole-brain-analyses as well as connectivity analyses.

Potential applications: As one example, out of the numerous publications based on fMRI methods, the publication by Mohnke and colleagues summarizes findings in individuals with pedophilic sexual interest (Mohnke et al., 2014). Their overview over seven studies resulted in some evidence for functional differences between individuals with and without pedophilic sexual interests in response to sexual stimuli, mainly for activity in the dorsal anterior cingulate while waiting for the stimulus presentation and activity in the hypothalamus during the actual stimulus presentation. At the time of their review findings were rather inconsistent and difficult to compare due to a high method variance, therefore the authors excluded the possibility of using functional activity data to differentiate individuals with from individuals without pedophilic sexual interests on an individual basis (Ponseti et al., 2012).

The fast-developing methods of analyses and growing knowledge about brain functionality suggests that activity in specific regions of the brain need to be considered in conjunction with other regions (network analyses) as well as in conjunction with connection between regions (connectivity analyses). An interesting 
example of the relevance of this combination is a study in high versus low psychopathic individuals exposed to facial expressions of different emotions. Contreras-Rodriguez and colleagues found a decreased functional connectivity between prefrontal and limbic-paralimbic structures as well as an increased connectivity within the dorsal frontal lobe linked to psychopathic personality (Contreras-Rodríguez et al., 2015). These data, in conjunction with their findings on facial expression processing (Contreras-Rodríguez et al., 2013), provides evidence for compensatory mechanisms based on these connectivity differences. Thus, using these functional imaging data, we can investigate not only static differences between groups in activity during processing of certain stimuli, but also investigate how differences between individuals might be related to interaction between brain areas, which can be targeted by potential treatment interventions.

\subsubsection{Positron-Emission-Tomography (PET)}

PET is another functional brain imaging method, however much less commonly used. The computer tomography detects pairs of gamma rays emitted by radioactive material that is injected or inhaled by the participant, such as oxygen, fluorine, carbon and nitrogen. Thus, the injected radioactive materials will be traced where they are used in the brain, where it will be broken down. The break-down of the radioactive material produced two gamma rays which can be imaged with the CT. Although PET has the potential to actually image activity of receptors and ligands, it is a very expensive and support-intensive method as the radioactive materials have to be produced on right before its use.

Potential applications: An example of an investigation using PET, in this case combined with fMRI is a study by Kolla and colleagues (2016), targeting the Monoamine oxidase A (MAO-A) levels in the ventral striatum. MAO-A is an enzyme that metabolizes monoamine neurotransmitters and has been found to be connected 
to impulsive aggression. In this study, the MAO-A total distribution volume in the ventral striatum was associated with functional connectivity of the inferior and superior ventral striatum in individuals with ASPD. Furthermore, these functional and structural measures were found to correlate with self-reported impulsivity, such as attentional and motor impulsivity. The authors claim this would imply that the low ventral striatum MAO-A levels might reflect the low Dopamine metabolism rate in the ventral striatum and thus explaining self-reported impulsive behaviour not only by low Dopamine levels but also reduced functional connectivity of striatal areas. This provides a good example of combining several imaging methods in order to learn not only about activity level differences but also putting them in context with brain connectivity and specific related neurotransmitter metabolism.

\subsection{Other relevant methods}

Methods that are less often used for research in forensic research so far, but which provide opportunities for understanding cognitive processes that might underlie antisocial behaviour are numerous. The following methods are particularly promising and are rapidly attracting interest from researchers. For the sake of completeness, lesion models are also included in this list. They are based on investigating individuals with specific brain injuries, with the most often cited example that of Phineas Gage case (Damasio, Grabowski, Frank, Galaburda, \& Damasio, 1994). As the lesions (in humans) are arbitrary, there is limited scope for investigating specific research questions. However, two methods are particularly valuable important for gaining a "holistic" understanding of brain functionality, especially with respect to complex phenomena like antisocial behaviour.

Transcranial Magnetic Stimulation (TMS) is a neuro-stimulation method for which an electromagnetic coil is placed on the participant's scalp, passing current through the scalp for about 1ms, peaking within $200 \mu$ s. This creates an electric 
current in the underlying brain tissue and this way disrupts the normal pattern of activity (Taylor, Walsh, \& Eimer, 2008). The repetitive induction of the magnetic stimulation with a certain frequency is called repetitive TMS (rTMS). The method allows to measure brain activity close to the scalp, but has specifically been used to measure cortical excitability, which is to what extent the brain can be activated. Further, the stimulation can be used to activate specific brain areas aiming at changing their activity over time.

As one example for an application in forensic psychology, a study used the stimulation to investigate cortical inhibition capacities in violent offenders compared to a control group (Philipp-Wiegmann et al., 2011). They used paired contralateral stimulation of the motor cortex, measuring the magnetically evoked potential provoked by the stimulation on the scalp. The authors found a reduced cortical inhibition in the left cortex of violent offenders and argue that this is related to the frontal dysfunction leading to low behaviour control. Thus, they found a direct correlate of offending behaviour in a neural measure that describes the direct the relationship between neural and behavioural activity.

Transcranial Direct Current Stimulation (tDCS) is a neuro-stimulation method that uses current passed from one electrode (anode) to another one (cathode). Anodal stimulation of the motor cortex increases neural excitability and cathodal stimulation decreases excitability (Filmer, Dux, \& Mattingley, 2014). Although the effects of the stimulation for depression (Shiozawa et al., 2014) and executive functioning (Horvath, Forte, \& Carter, 2015a, 2015b) are not established, the method can lead to improvements in cognitive and memory tasks in elderly (Summers, Kang, \& Cauraugh, 2016).

Near Infrared Spectroscopy (NIRS) is a method that uses infrared light to measure oxygenated and deoxygenated haemoglobin in a less resource consuming 
way compared to fMRI (Lloyd-Fox, Blasi, \& Elwell, 2010; Plichta et al., 2006). There has been limited use of the method for forensic research questions so far. However, due to the less cost-intense administration compared to MRI assessments, this method might become more relevant for use with criminal populations in the future.

\section{Challenges for neuroscientific approaches in forensic psychology}

There are several challenges to the use of neuroscientific methods for questions in forensic psychology, some of which can and are currently addressed (also see Ward et al., this issue). One hurdle to the use of such methods in forensic contexts is the accessibility of technical equipment specifically for incarcerated populations. Researchers sometimes struggle with institutional regulations for taking equipment such as EEG or NIRS into prisons or forensic hospitals or bringing patients or prisoners out of the facilities to research centres, depending on local and national regulations. The emergence of mobile MRI equipment has contributed positively to an increased intramural access to imaging equipment (Abbott, 2007).

However, more important than the access to participants is the data quality, which depends heavily on trained researchers and staff, more specifically regarding careful designing experimental studies and use of assessment tools, as well as the interpretation of the results. It will be important to continue good training for future researchers and to engage cognitive neuroscientists in collaborations with forensic psychologists.

Another complication that is specific to samples with a history of antisocial behaviour is an increased rate of exclusion of potential study participants based on method-based exclusion criteria. Participation in an MRI based study is restricted to individuals without recent tattoos, without a history of working in metalworking industry and without medical implants. Although, as these restrictions do not apply 
to all neuroscientific methods, its arguably has a rather minor effect in terms of recruitment bias.

One of the less technical challenges might lie in the use and misuse of those methods on an individual basis for behaviour prediction, so called Neuroprediction. Neurophysiological data can be used in approaches that try to link individual neurophysiological responses or characteristics to predict future behaviour. In the case of forensic psychology, the question about future criminal behaviour both in terms of recidivism and in terms of potential biomarkers indicating individuals atrisk without prior offenses is a highly sensitive area. Studies that show a high predictive accuracy to detect, for example, sexual interests based on functional cortical activity in response to sexual stimuli (Ponseti et al., 2012) or completion of a substance abuse treatment program based on functional reactivity in a cognitive task (Steele et al., 2014), are important moving forward in order to understand the relevance of cognitive neuroscience findings for actual behaviour. However, the use of those measures in the courtroom is controversial (see e.g., Meynen, 2016).

Related to the neuroprediction challenge is the question about the validity of the use of neuroimaging data in the court room. As studies have shown that participants in the trial like jury members can be influenced by the use of presentation of neuroscientific evidence (e.g., McCabe, Castel, \& Rhodes, 2011), it is very important for expert witnesses to know about those methods and their potential of application (J ones, Wagner, Faigman, \& Raichle, 2013). However, the influence of presenting neuroscientific evidence is just on top of a more fundamental problem: apart from structural brain abnormalities which are individual-specific and can be included as evidence for behavioural abnormalities (see Eastman \& Campbell, 2006), findings from group based studies such as those presented in this review can not yet be used on individual basis, i.e. measuring the $\mathrm{P} 3$ in response to a Go/Nogo task in a 
single individual cannot be used for diagnostic purposes. Although the abovementioned study that differentiated juvenile offenders for low and high scores of psychopathic traits based on structural brain data (Steele et al., 2017), the accuracy is not high enough and more importantly, the variability based on methods of measurement and analysis (pre-processing of the data) is too high to be a sufficient basis for individual cases. However, careful use might be helpful to describe underlying neural abnormalities as part of a wider assessment. Another application of neuroimaging methods that gets a lot of attention in clinical psychology and psychiatry is the prediction of treatment outcomes based on neural structure or functionality. E.g, a recent study showed that researchers were able to differentiate substance abuse treatment outcomes based on functional connectivity data (Steele et al., in press). Thus they could predict if a participant in from a treatment group finished the program or not by functional connectivity data related to frontal and limbic areas of the brain (see Figure 5). As treatment programs are expensive and should be well-tailored to needs and acceptance, this type of predictive assessments might become more relevance in the future.

\section{Conclusions}

This review aimed at providing a quick overview of neuroscientific methods with relevance for forensic psychology. As these methods will become more important for assessments and for the understanding of criminal behaviour, knowledge and ability to interpret such findings is crucial for forensic psychologists. As those methods advance more and more rapidly (Stevenson \& Kording, 2011), it is and will make it hard to keep up with the developments and estimations of their validity. However, as challenges are arguably more advantageous than detrimental in development of science (DePasque Swanson \& Tricomi, 2014), these aforementioned 
ones will be addressed and increase the use of cognitive neuroscience methods in forensic psychology. 


\section{References}

Abbott, A. (2007). Abnormal neuroscience: Scanning psychopaths Nature (Vol. 450, pp. 942944).

Alexander, A. L., Lee, J. E., Lazar, M., \& Field, A. S. (2007). Diffusion Tensor Imaging of the Brain. Neurotherapeutics : the journal of the American Society for Experimental NeuroTherapeutics, 4(3), 316-329. doi: 10.1016/j.nurt.2007.05.011

Barham, M. P., Clark, G. M., Hayden, M. J., Enticott, P. G., Conduit, R., \& Lum, J. A. G. (2017). Acquiring research-grade ERPs on a shoestring budget: A comparison of a modified Emotiv and commercial SynAmps EEG system. Psychophysiology, 54(9), 1393-1404. doi: 10.1111/psyp.12888

Bigler, E. D. (2015). Structural Image Analysis of the Brain in Neuropsychology Using Magnetic Resonance Imaging (MRI) Techniques. [journal article]. Neuropsychology Review, 25(3), 224-249. doi: 10.1007/s11065-015-9290-0

Calhoun, Vince D., Adali, T., Pearlson, G. D., \& Pekar, J. J. (2001). A method for making group inferences from functional MRI data using independent component analysis. Human Brain Mapping, 14, 140-151.

Choi, O. S. (2017). What Neuroscience Can and Cannot Answer. The Journal of the American Academy of Psychiatry and the Law, 45(3), 278-285.

Contreras-Rodríguez, O., Pujol, J., Batalla, I., Harrison, B. J., Bosque, J., Ibern-Regàs, I., . . . Cardoner, N. (2013). Disrupted neural processing of emotional faces in psychopathy. Social Cognitive and Affective Neuroscience. doi: 10.1093/scan/nst014

Contreras-Rodríguez, O., Pujol, J., Batalla, I., Harrison, B. J., Soriano-Mas, C., Deus, J., . . . Cardoner, N. (2015). Functional Connectivity Bias in the Prefrontal Cortex of Psychopaths. Biological Psychiatry, 78(9), 647-655. doi: 10.1016/j.biopsych.2014.03.007 
Damasio, H., Grabowski, T., Frank, R., Galaburda, A. M., \& Damasio, A. R. (1994). The return of Phineas Gage: clues about the brain from the skull of a famous patient. Science, 264(5162), 1102-1105.

DePasque Swanson, S., \& Tricomi, E. (2014). Goals and task difficulty expectations modulate striatal responses to feedback. Cognitive, Affective \& Behavioral Neuroscience, 14(2), 610-620. doi: 10.3758/s13415-014-0269-8

Eastman, N., \& Campbell, C. (2006). Neuroscience and legal determination of criminal responsibility. Nature Reviews Neuroscience, 7(4), 311-318.

Filmer, H. L., Dux, P. E., \& Mattingley, J. B. (2014). Applications of transcranial direct current stimulation for understanding brain function. Trends in Neurosciences, 37(12), 742-753. doi: 10.1016/j.tins.2014.08.003

Fisher, S. F. (2007). Neurofeedback, affect regulation and attachment: A case study and analysis of anti-social personality. International Journal of Behavioral Consultation and Therapy, 3(1), 109-117. doi: 10.1037/h0100171

Gao, Y., \& Raine, A. (2009). P3 event-related potential impairments in antisocial and psychopathic individuals: A meta-analysis. Biological Psychology, 82(3), 199-210. doi: 10.1016/j.biopsycho.2009.06.006

Gao, Y., Zhang, W., Eisenbarth, H., Lai-Chu Fung, A., Lu, M., Raine, A., \& Li, X. (2018). P3 amplitude and psychopathic traits in youths: Distinct contributions of the grandiose-manipulative and daring-impulsivity traits. Personality and Individual Differences, 120(1), 87-94. doi: 10.1016/j.paid.2017.08.017

Glenn, A. L., \& Raine, A. (2014). Neurocriminology: implications for the punishment, prediction and prevention of criminal behaviour. [Perspectives]. Nat Rev Neurosci, 15(1), 54-63. doi: 10.1038/nrn3640 
Harmon-Jones, E., Gable, P. A., \& Peterson, C. K. (2010). The role of asymmetric frontal cortical activity in emotion-related phenomena: a review and update. Biol Psychol, 84(3), 451-462. doi: 10.1016/j.biopsycho.2009.08.010

Horvath, J. C., Forte, J. D., \& Carter, O. (2015a). Evidence that transcranial direct current stimulation (tDCS) generates little-to-no reliable neurophysiologic effect beyond MEP amplitude modulation in healthy human subjects: A systematic review. Neuropsychologia, 66(Supplement C), 213-236. doi:

10.1016/j.neuropsychologia.2014.11.021

Horvath, J. C., Forte, J. D., \& Carter, O. (2015b). Quantitative Review Finds No Evidence of Cognitive Effects in Healthy Populations From Single-session Transcranial Direct Current Stimulation (tDCS). Brain Stimulation, 8(3), 535-550. doi:

10.1016/j.brs.2015.01.400

Jones, O. D., Wagner, A. D., Faigman, D. L., \& Raichle, M. E. (2013). Neuroscientists in court. Nature Reviews Neuroscience. 14(10), 730-736. doi: 10.1038/nrn3585

Keil, A., Debener, S., Gratton, G., Junghöfer, M., Kappenman, E. S., Luck, S. J., . . Yee, C. M. (2013). Committee report: Publication guidelines and recommendations for studies using electroencephalography and magnetoencephalography. Psychophysiology, 51(1), 1-21. doi: 10.1111/psyp.12147

Kolla, N. J., Dunlop, K., Downar, J., Links, P., Bagby, R. M., Wilson, A. A., . . Meyer, J. H. (2016). Association of ventral striatum monoamine oxidase-A binding and functional connectivity in antisocial personality disorder with high impulsivity: A positron emission tomography and functional magnetic resonance imaging study. Eur Neuropsychopharmacol, 26(4), 777-786. doi: 10.1016/j.euroneuro.2015.12.030 
Konicar, L., Veit, R., Eisenbarth, H., Barth, B., Tonin, P., Strehl, U., \& Birbaumer, N. (2015). Brain self-regulation in criminal psychopaths. [Article]. Scientific Reports, 5. doi: 10.1038/srep09426

Lake, S. L., Stanford, M. S., \& Patton, J. H. (2014). Emotional processing and frontal asymmetry in impulsive aggressive individuals. Personality and Individual Differences, 70(0), 131-135. doi: 10.1016/j.paid.2014.06.025

Lamsma, J., Mackay, C., \& Fazel, S. (2017). Structural brain correlates of interpersonal violence: Systematic review and voxel-based meta-analysis of neuroimaging studies. Psychiatry Res, 267, 69-73. doi: 10.1016/j.pscychresns.2017.07.006

Lloyd-Fox, S., Blasi, A., \& Elwell, C. E. (2010). Illuminating the developing brain: The past, present and future of functional near infrared spectroscopy. Neuroscience \& Biobehavioral Reviews, 34(3), 269-284. doi: 10.1016/j.neubiorev.2009.07.008

McCabe, D. P., Castel, A. D., \& Rhodes, M. G. (2011). The influence of fMRI lie detection evidence on juror Decision-Making. Behavioral sciences \& the law, 29(4), 566-577.

Meynen, G. (2016). Neurolaw: recognizing opportunities and challenges for psychiatry. Journal of Psychiatry \& Neuroscience, 41(1), 3-5. doi: 10.1503/jpn.150317

Mohnke, S., Muller, S., Amelung, T., Kruger, T. H., Ponseti, J., Schiffer, B., . . Walter, H. (2014). Brain alterations in paedophilia: a critical review. Prog Neurobiol, 122, 1-23. doi: 10.1016/j.pneurobio.2014.07.005

Neal, L. B., \& Gable, P. A. (2017). Regulatory control and impulsivity relate to resting frontal activity. Soc Cogn Affect Neurosci, 12(9), 1377-1383. doi: 10.1093/scan/nsx080

Oliver, T. L., Meana, M., \& Snyder, J. S. (2016). Sex differences in concordance rates between auditory event-related potentials and subjective sexual arousal. Psychophysiology, 53(8), 1272-1281. doi: 10.1111/psyp.12661 
Olvet, D. M., \& Hajcak, G. (2009). Reliability of error-related brain activity. Brain Research, 1284, 89-99.

Oostermeijer, S., Whittle, S., Suo, C., Allen, N. B., Simmons, J. G., Vijayakumar, N., . . . Popma, A. (2016). Trajectories of adolescent conduct problems in relation to cortical thickness development: a longitudinal MRI study. Transl Psychiatry, 6(6), e841. doi: 10.1038/tp.2016.111

Philipp-Wiegmann, F., Roesler, M., Roemer, K. D., Schneider, M., Baumgart, S., \& Retz, W. (2011). Reduced Cortical Inhibition in Violent Offenders: A Study with Transcranial Magnetic Stimulation. Neuropsychobiology, 64(2), 86-92. doi:10.1159/000326694

Picton, T., Bentin, S., Berg, P., Donchin, E., Hillyard, S., Johnson, R., . . . Taylor, M. (2000). Guidelines for using human event-related potentials to study cognition: Recording standards and publication criteria. Psychophysiology, 37(2), 127-152.

Plichta, M. M., Herrmann, M. J., Baehne, C. G., Ehlis, A. C., Richter, M. M., Pauli, P., \& Fallgatter, A. J. (2006). Event-related functional near-infrared spectroscopy (fNIRS): Are the measurements reliable? NeuroImage, 31(1), 116-124. doi: 10.1016/j.neuroimage.2005.12.008

Ponseti, J., Granert, O., Jansen, O., Wolff, S., Beier, K., Neutze, J., . . Bosinski, H. (2012). Assessment of Pedophilia Using Hemodynamic Brain Response to Sexual Stimuli. Archives of General Psychiatry, 69(2), 187-194. doi:

10.1001/archgenpsychiatry.2011.130

Prause, N., Staley, C., \& Roberts, V. (2014). Frontal alpha asymmetry and sexually motivated states. Psychophysiology, 51(3), 226-235. doi: 10.1111/psyp.12173

Prause, N., Steele, V. R., Staley, C., \& Sabatinelli, D. (2015). Late positive potential to explicit sexual images associated with the number of sexual intercourse partners. 
Social Cognitive and Affective Neuroscience, 10(1), 93-100. doi:

\subsection{3/scan/nsu024}

Reyes, A. C., \& Amador, A. A. (2009). Qualitative and quantitative EEG abnormalities in violent offenders with antisocial personality disorder. J Forensic Leg Med, 16(2), 5963. doi: 10.1016/j.jflm.2008.08.001

Schupp, H. T., Flaisch, T., Stockburger, J., \& Junghoefer, M. (2006). Emotion and attention: event-related brain potential studies. Progress in Brain Research, 156, 31-51.

Shiozawa, P., Fregni, F., Benseñor, I. M., Lotufo, P. A., Berlim, M. T., Daskalakis, J. Z., .. . Brunoni, A. R. (2014). Transcranial direct current stimulation for major depression: an updated systematic review and meta-analysis. International Journal of Neuropsychopharmacology, 17(9), 1443-1452. doi: 10.1017/S1461145714000807

Steele, V. R., Fink, B. C., Maurer, J. M., Arbabshirani, M. R., Wilber, C. H., Jaffe, A. J., .. . Kiehl, K. A. (2014). Brain potentials measured during a Go/NoGo task predict completion of substance abuse treatment. Biol Psychiatry, 76(1), 75-83. doi: 10.1016/j.biopsych.2013.09.030

Steele, V. R., Maurer, J. M., Arbabshirani, M. R., Claus, E. D., Fink, B. C., Rao, V., ... Kiehl, K. A. (in press). Machine Learning of Functional Magnetic Resonance Imaging Network Connectivity Predicts Substance Abuse Treatment Completion. Biological Psychiatry: Cognitive Neuroscience and Neuroimaging. doi: 10.1016/j.bpsc.2017.07.003

Steele, V. R., Rao, V., Calhoun, V. D., \& Kiehl, K. A. (2017). Machine learning of structural magnetic resonance imaging predicts psychopathic traits in adolescent offenders. Neuroimage, 145(Pt B), 265-273. doi: 10.1016/j.neuroimage.2015.12.013

Stevenson, I. H., \& Kording, K. P. (2011). How advances in neural recording affect data analysis. Nature Neuroscience, 14, 139-142. doi: 10.1038/nn.2731 
Summers, J. J., Kang, N., \& Cauraugh, J. H. (2016). Does transcranial direct current stimulation enhance cognitive and motor functions in the ageing brain? A systematic review and meta- analysis. Ageing Research Reviews, 25(Supplement C), 42-54. doi: 10.1016/j.arr.2015.11.004

Taylor, P. C. J., Walsh, V., \& Eimer, M. (2008). Combining TMS and EEG to study cognitive function and cortico-cortico interactions. Behavioural Brain Research, 191(2), 141-147.

Waller, R., Dotterer, H. L., Murray, L., Maxwell, A. M., \& Hyde, L. W. (2017). White-matter tract abnormalities and antisocial behavior: A systematic review of diffusion tensor imaging studies across development. Neuroimage Clin, 14, 201-215. doi: 10.1016/j.nicl.2017.01.014

Woodman, G. F. (2010). A Brief Introduction to the Use of Event-Related Potentials (ERPs) in Studies of Perception and Attention. Attention, perception \& psychophysics, 72(8), 10.3758/APP.3772.3758.2031. doi: 10.3758/APP.72.8.2031

Zhong, W., Ciatipis, M., Wolfenstetter, T., Jessberger, J., Müller, C., Ponsel, S., . . Draguhn, A. (2017). Selective entrainment of gamma subbands by different slow network oscillations Proceedings of the National Academy of Sciences of the United States of America, 114(7), 4519-4524. doi: 10.1073/pnas.1617249114 
Tables and Figures

Table 1: Structural and functional neuroimaging techniques overview

\begin{tabular}{|c|c|c|c|c|}
\hline Measurement & Signal & Neurophysiological process & $\begin{array}{l}\text { Time } \\
\text { resolution }\end{array}$ & $\begin{array}{l}\text { Spatial } \\
\text { resolution }\end{array}$ \\
\hline VBM & Metabolic & $\begin{array}{l}\text { No process, visualization of } \\
\text { grey versus white matter }\end{array}$ & - & \\
\hline DTI & Metabolic & $\begin{array}{l}\text { Functional integrity of nerve } \\
\text { bundles } \\
\text { Summation of frequency }\end{array}$ & & high \\
\hline EEG & Electrical & $\begin{array}{l}\text { oscillations based on electrical } \\
\text { signal }\end{array}$ & high & low \\
\hline fMRI & Metabolic & Oxygen saturation within & low & high \\
\hline PET & Metabolic & $\begin{array}{l}\text { Metabolic activity rates in } \\
\text { groups of neurons }\end{array}$ & low & high \\
\hline MEG & Electrical & & low & medium \\
\hline
\end{tabular}


Table 2: Event-related Potentials (ERPs) and linked processes

$\begin{array}{ll}\text { ERP Description } & \text { Time after stimulus- Processes } \\ \text { onset }\end{array}$

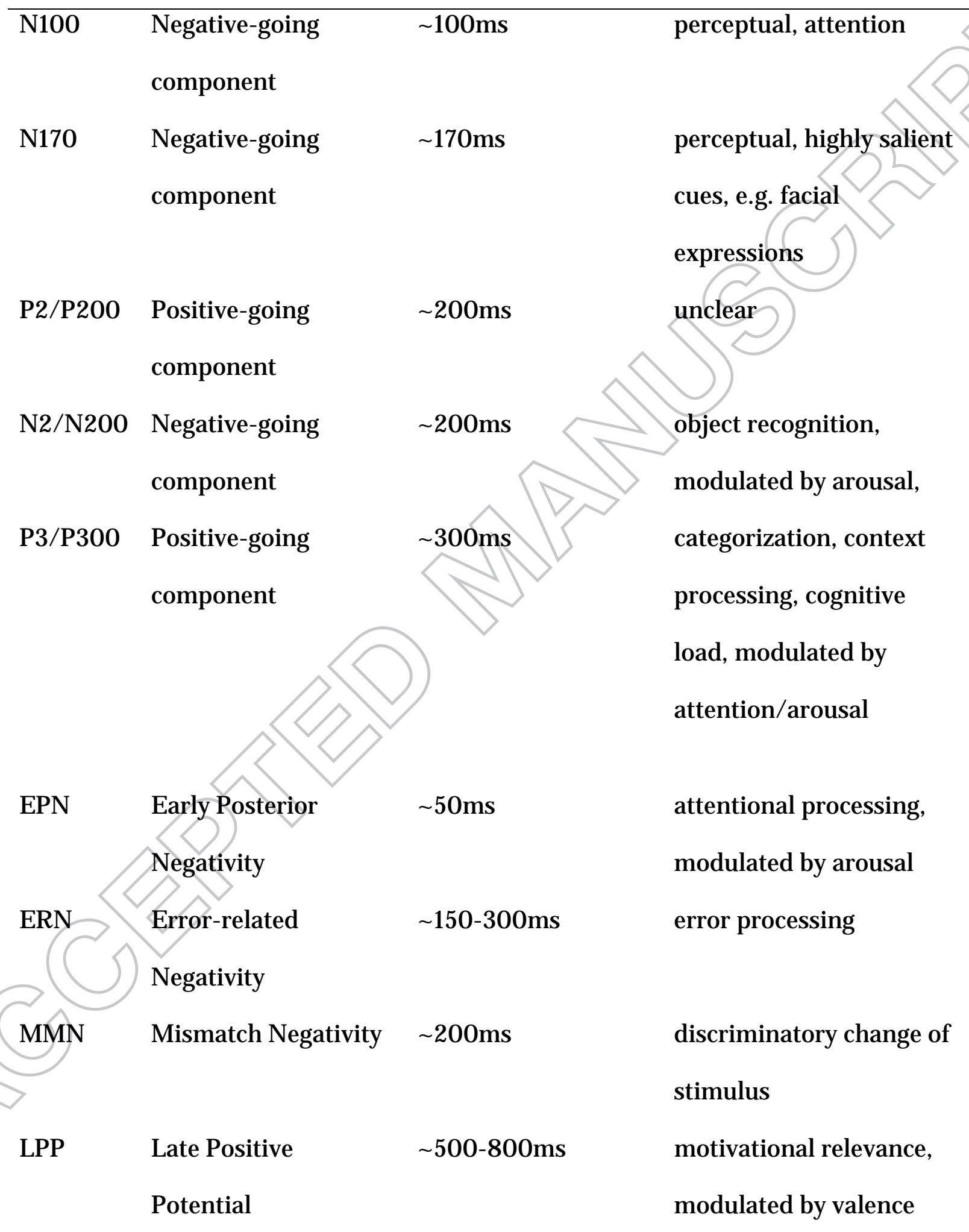



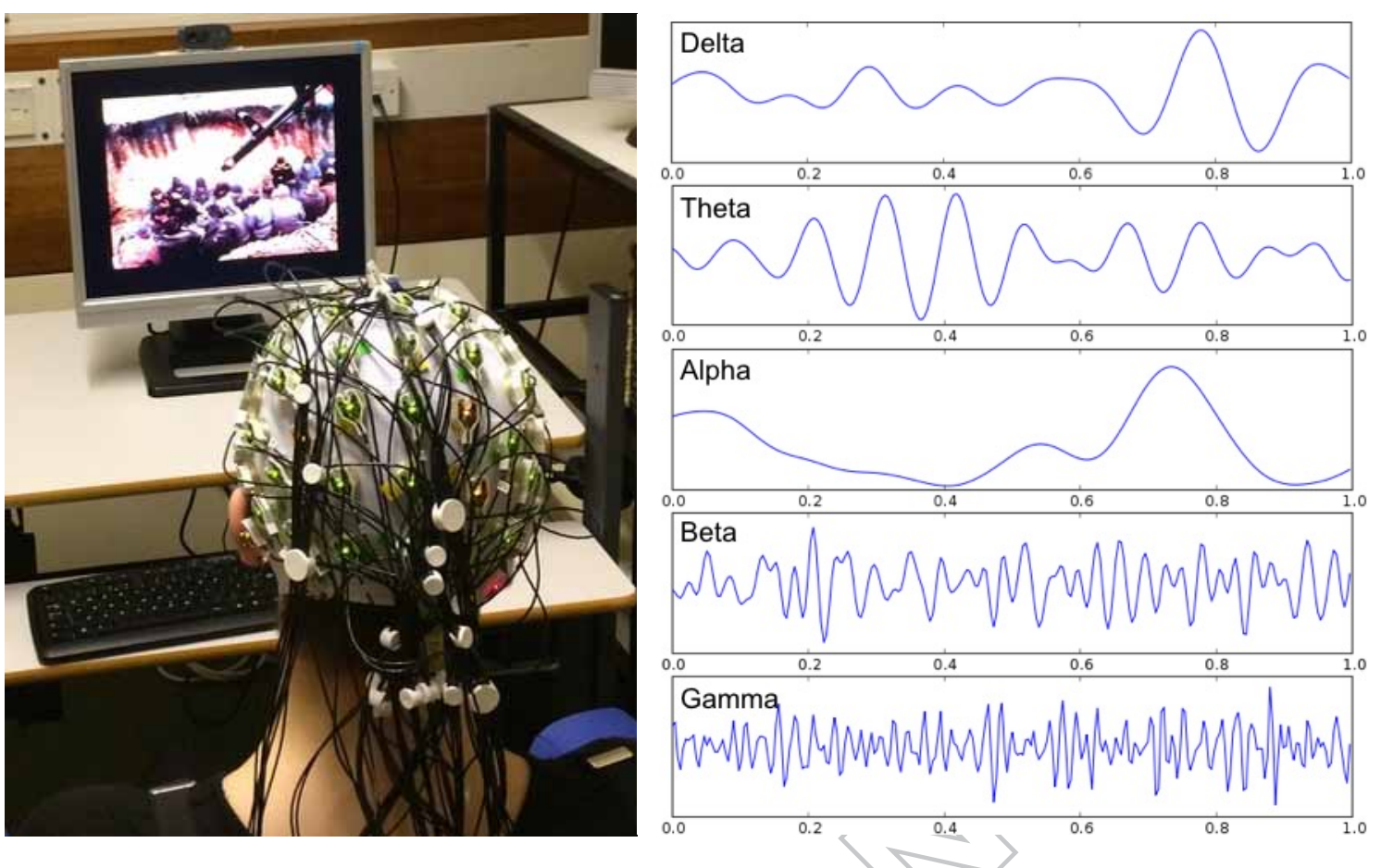

Figure 1: Set-up of a participant with EEG and an overview of EEG frequency bands 


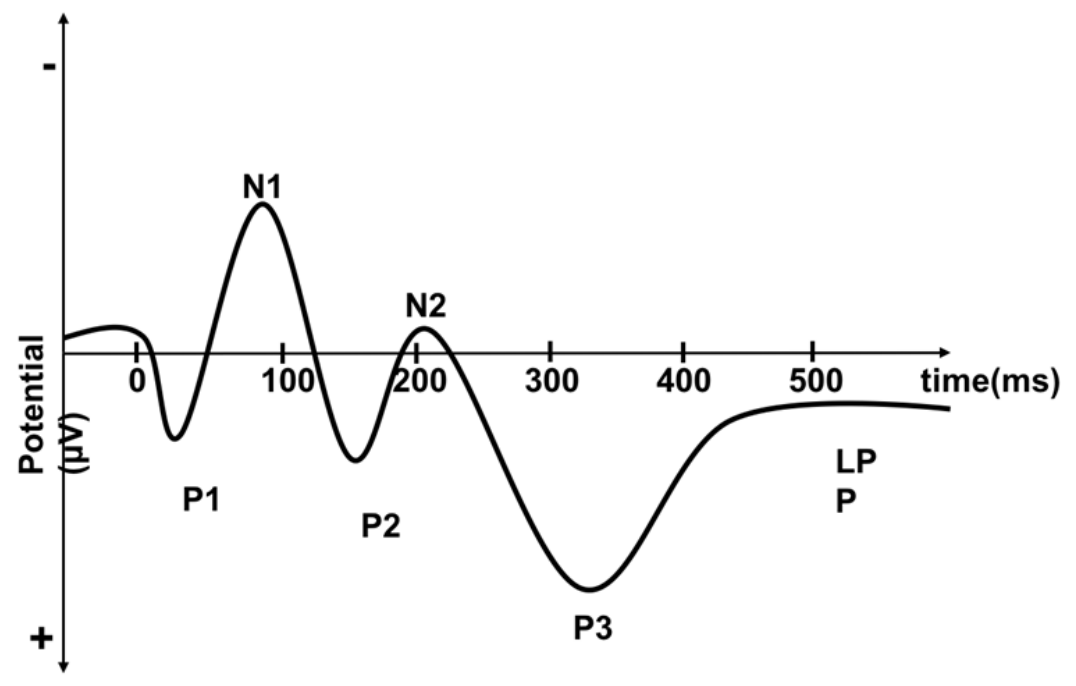

Figure 2: Schematic representation of event related potentials (ERP) over time; caveat: potentials can be presented either way: showing negativity upwards (as in this representation) or showing negativity downwards. 


\section{- PCL-R $>20 \quad$ PCL-R $<20$}

\section{Fearful Faces}

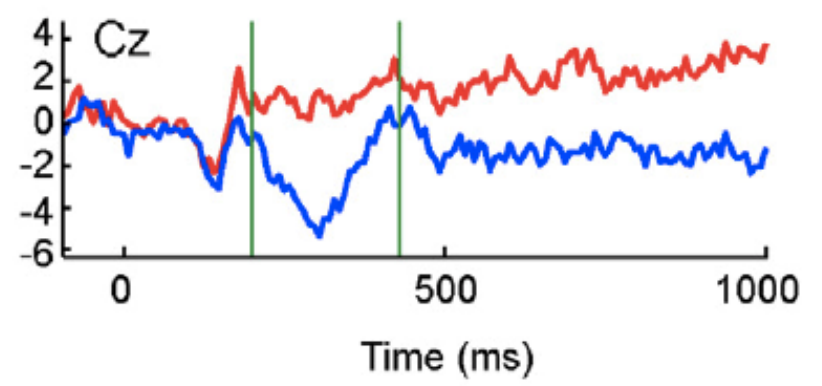

Figure 3: Event-related potential after presentation of fearful facial expressions comparing a group of highly psychopathic female offenders (red) with a group of low psychopathic female offenders (blue), showing a reduced N2 (between the two green lines) in the former group (Reprinted from Biological Psychology, 94(2), Eisenbarth, H., Angrilli, A., Calogero, A., Harper, J., Olson, L. A., \& Bernat, E., Reduced negative affect response in female psychopaths, Figure 2, page 314, Copyright (2013), with permission from Elsevier). 


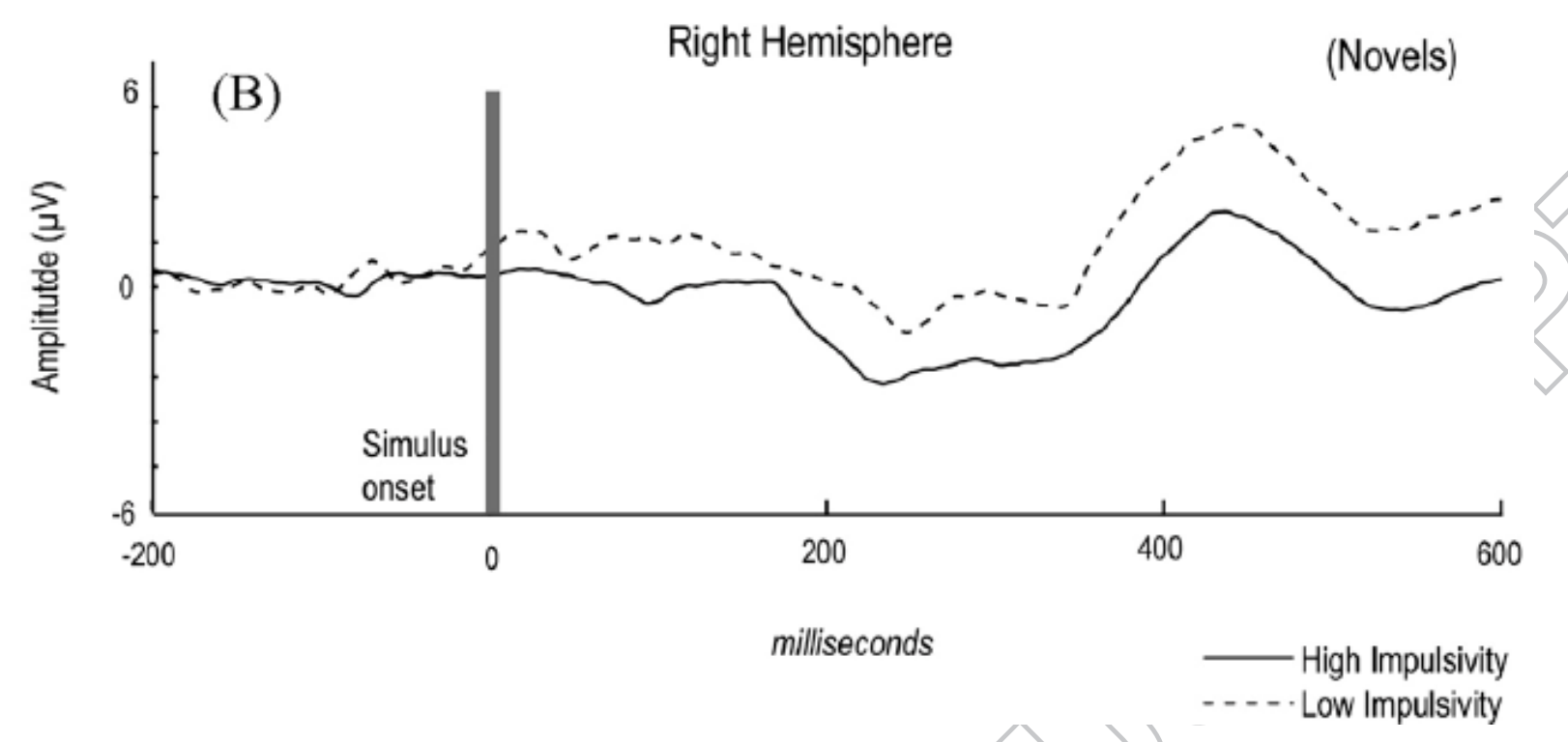

Figure 4: ERP (P3) for right hemisphere activity in response to novel stimuli in a Go/ Nogo task, comparing youth high and low on the Impulsivity aspect of callousunemotional traits, visible in the window between 300 and 500ms (Reprinted from Personality and Individual Differences, 120, Gao, Y., Zhang, W., Eisenbarth, H., Fung, A. L.-C., Lu, M., Raine, A., Leeh, T. M.C. \& Lig, X., P3 amplitude and psychopathic traits in youths: Distinct contributions of the grandiose-manipulative and daring-impulsivity traits, Figure 2, page 91, Copyright (2018), with permission from Elsevier). 

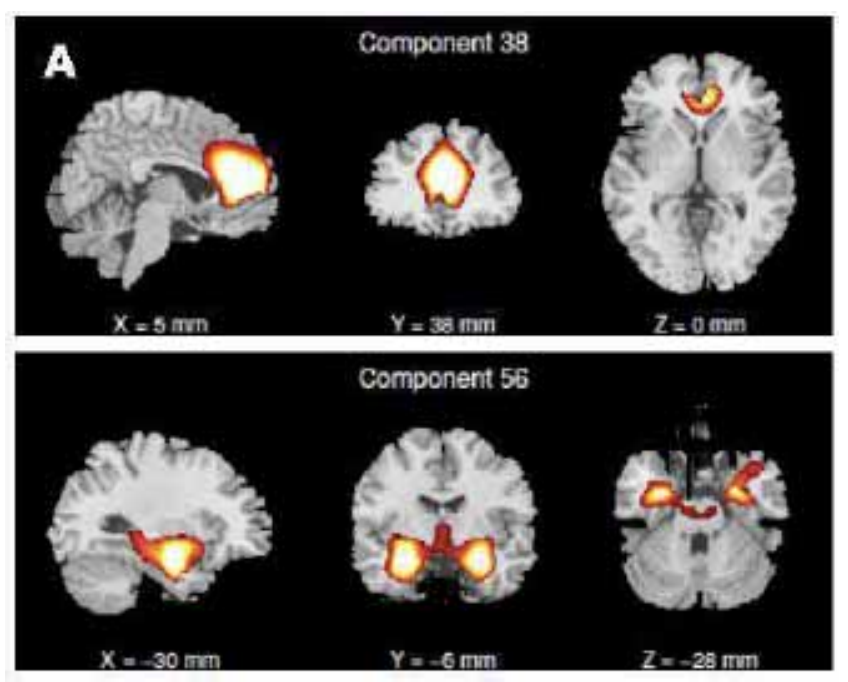

Figure 5: Stronger functional network connectivity differentiating completion of treatment versus discontinuation; e.g. connectivity between rostral anterior cingulate cortex (rACC, component 38) and component 56, including the amygdala, hippocampus, and striatum (Reprinted from Biological Psychiatry: Cognitive Neuroscience and Neuroimaging, in press, Steele, V. R., Maurer, J . M., Arbabshirani, M. R., Claus, E. D., Fink, B. C., Rao, V., Calhoun, V. D. \& Kiehl, K. A., Machine Learning of Functional Magnetic Resonance Imaging Network Connectivity Predicts Substance Abuse Treatment Completion, Figure 2, page tbd, Copyright (2018), with permission from Elsevier). 